\title{
Integrative analyses of targeted metabolome and transcriptome of Isatidis Radix autotetraploids highlighted key polyploidization-responsive regulators
}

Zixuan Zhang ${ }^{1 \dagger}$, Mingpu Tan ${ }^{2 \dagger}$, Yingying Zhang ${ }^{2}$, Yue Jia', Shuxian Zhu' ${ }^{2}$, Jiang Wang ${ }^{1}$, Jiajing Zhao', Yueyue Liao ${ }^{1}$ and Zengxu Xiang ${ }^{1^{*}}$

\begin{abstract}
Background: Isatidis Radix, the root of Isatis indigotica Fort. (Chinese woad) can produce a variety of efficacious compound with medicinal properties. The tetraploid I. indigotica plants exhibit superior phenotypic traits, such as greater yield, higher bioactive compounds accumulation and enhanced stress tolerance. In this study, a comparative transcriptomic and metabolomic study on Isatidis Radix autotetraploid and its progenitor was performed.

Results: Through the targeted metabolic profiling, 283 metabolites were identified in Isatidis Radix, and 70 polyploidization-altered metabolites were obtained. Moreover, the production of lignans was significantly increased post polyploidization, which implied that polyploidization-modulated changes in lignan biosynthesis. Regarding the transcriptomic shift, 2065 differentially expressed genes (DEGs) were identified as being polyploidy-responsive genes, and the polyploidization-altered DEGs were enriched in phenylpropanoid biosynthesis and plant hormone signal transduction. The further integrative analysis of polyploidy-responsive metabolome and transcriptome showed that 1584 DEGs were highly correlated with the 70 polyploidization-altered metabolites, and the transcriptional factors TFs-lignans network highlighted 10 polyploidy-altered TFs and 17 fluctuated phenylpropanoid pathway compounds.

Conclusions: These results collectively indicated that polyploidization contributed to the high content of active compounds in autotetraploid roots, and the gene-lignan pathway network analysis highlighted polyploidyresponsive key functional genes and regulators.
\end{abstract}

Keywords: Polyploidization, Metabolome, Transcriptional factors, lignan biosynthesis, Isatidis Radix

\footnotetext{
*Correspondence: zxxiang@njau.edu.cn

†Zixuan Zhang and Mingpu Tan contributed equally to this work.

${ }^{1}$ College of Horticulture, Nanjing Agricultural University, 210095 Nanjing,

China

Full list of author information is available at the end of the article
}

C C The Author(s). 2021 Open Access This article is licensed under a Creative Commons Attribution 4.0 International License, which permits use, sharing, adaptation, distribution and reproduction in any medium or format, as long as you give appropriate credit to the original author(s) and the source, provide a link to the Creative Commons licence, and indicate if changes were made. The images or other third party material in this article are included in the article's Creative Commons licence, unless indicated otherwise in a credit line to the material. If material is not included in the article's Creative Commons licence and your intended use is not permitted by statutory regulation or exceeds the permitted use, you will need to obtain permission directly from the copyright holder. To view a copy of this licence, visit http://creativecommons.org/licenses/by/4.0/ The Creative Commons Public Domain Dedication waiver (http://creativecommons.org/publicdomain/zero/1.0/) applies to the data made available in this article, unless otherwise stated in a credit line to the data. 


\section{Introduction}

Isatis indigotica Fort. (Chinese woad, $2 n=14$ ) belongs to Isatideae tribe of the Brassicaceae family. The root of I. indigotica called Isatidis Radix, which can produce a variety of chemicals with medicinal properties, can be used in clinical treatment of regular seasonal influenza and plays an immune regulatory role in vitro and in vivo [1], while the leaves of I. indigotica called Isatidis Folium composed of isatin, tryptanthrin, indirubin and so on [2,3]. Several categories of metabolites including alkaloids, phenylpropanol, organic acids and polysaccharides identified from Isatidis Radix, were demonstrated to achieve the antiviral and antioxidant effects [4-6]. Therefore, increasing the abundance of the active compounds is critical for improving the quality of Isatidis Radix [7].

Polyploidy is widespread in plants, and nearly $70 \%$ of angiosperms are polyploids including many important crops [8]. Polyploidization, also known as whole genome duplication (WGD), plays a pivotal role in promoting the evolution of plant morphological, physiological and reproductive diversity [8-12]. Compared with their diploid progenitors, polyploid plants often exhibit superior phenotypic traits, such as stronger tolerance, higher content of active compounds, and enlarged organs together with increased vigor $[8,13-16]$. The most conspicuous features of polyploidy are the increased cell size, slowed cell division and tissue development, and increased organ size at maturity, which is referred to as the 'gigas effect' [11, 15]. The tetraploid I. indigotica accumulate more lignans than diploid, including lariciresinol and its derivatives, which present effective antiviral ingredients of $I$. indigotica [17]. The giant organs and enhanced concentrations of secondary metabolites realized by autopolyploidy are attractive for breeding the respective medicinal and agricultural plants.

However, there has been no report on the metabolomic and transcriptomic changes post polyploidization of Isatidis Radix until now. In the past several years, the research in the field of polyploidy is mainly focused on the transcriptional level, using RNAseq-based transcriptomic analysis to reveal the relationship between polyploidization and gene expression [16]. At present, only two reports of the Chinese Woad leaf (Isatidis Folium) transcriptomic changes induced by autotetraploidization were available [18, 19]. However, the root (Isatidis Radix) differed from leaf (Isatidis Folium) whether in biological function or in medicinal usage. And there are three monographs of $I$. indigotica included in the Chinese Pharmacopoeia, namely Isatidis Folium, Indigo Naturalis and Isatidis Radix [20], so these three kinds of Chinese herbal medicine preparations are somewhat different. Moreover, the metabolome is closer to phenotype than transcriptome.
Given that the metabolic activity was altered by the fluctuated gene expression, which led to the change of the concentration of secondary metabolites, we carried out a comparative transcriptomic and metabolomic study on Isatidis Radix autotetraploid and its progenitor. Through the integrative analysis of Isatidis Radix transcriptome and metabolome, the differentially expressed genes affecting the metabolic pathway of active components such as lignan were identified. As a result, the gene-lignan pathway network analysis highlighted polyploidy-responsive key functional genes and regulators.

\section{Materials and methods}

\section{Plant materials and sampling}

Appropriate permissions for collection and use of seed of Isatis indigotica Fort. $(2 n=2 x=14)$ was obtained from Jiangsu Germplasm Repository Center. I. indigotica $(2 n=2 x=14)$ used as diploid donor. Autotetraploid $I$. indigotica was artificially synthesized by colchicinemediated polyploidy induction in vitro as described previously $[16,19]$. Briefly, adventitious buds induced from diploid planet were subjected to $0.20 \%$ colchicine treatment for $12 \mathrm{~h}$, and transferred to MS medium without colchicine for 2 weeks. Then, the synthesized autotetraploid plantlets were transferred to $1 / 2 \mathrm{MS}$ medium for rooting. The root tips $(0.5-1 \mathrm{~cm}$ long) were excised and pretreated with $2 \mathrm{mmol} \cdot \mathrm{L}^{-1} 8$-hydroxyquinoline solution for $4 \mathrm{~h}$, and fixed with Carnoy's solution at $4{ }^{\circ} \mathrm{C}$ for $24 \mathrm{~h}$. Samples were then hydrolyzed using $1 \mathrm{~mol} \cdot \mathrm{L}^{-1}$ $\mathrm{HCl}$ at $60{ }^{\circ} \mathrm{C}$ for $10 \mathrm{~min}$. The hydrolyzed root tips were soaked in a drop of Carbol fuchsin for $10 \mathrm{~min}$ and squashed on the microscopic slide to observe the metaphase chromosomes. Finally, seedlings with roots were transplanted into nutritional soil. The diploid and autotetraploid I. indigotica seedlings were planted in the experimental fields in our campus for 1-year. Then, their roots were sampled for the subsequent transcriptomic and metabolomic analysis with three repeats (Fig.S1). The sampled fresh roots of I. indigotica were frozen with liquid nitrogen, transported and stored at $-80{ }^{\circ} \mathrm{C}$.

\section{Targeted metabolomic analysis of Isatidis Radix metabolites}

The Isatidis Radix samples were freeze-dried and ground into fine powder for metabonomic analysis. The widely targeted metabolic profile and quantitative detection of metabolites were performed by MetWare Biotechnology Co.,Ltd (Wuhan, China) (www.metware.cn). The quantification of metabolites was carried out using a predetermined multi-reaction monitoring method [21].

The elemental composition and mass fragmentation were compared to those registered inaccessible databases of NIST as well as the standards in a database compiled by MetWare Biotechnology Co.,Ltd [22]. 


\section{RNAseq libraries preparation and sequencing}

Total RNA for RNAseq was extracted from seedling roots and about $1 \mu \mathrm{g}$ RNA per sample was used as input material for the RNA sample preparations. Sequencing libraries were generated using $\mathrm{NEBNext}^{\circ}$ UltraTM RNA Library Prep Kit for Illumina ${ }^{\circ}$ (NEB, USA) and index codes were added to attribute sequences to each sample. For high-throughput sequencing, the library preparations were sequenced on an Illumina Hiseq X Ten platform and 150 bp pairedend reads were generated [19]. After the adaptor and low-quality sequences were trimmed, a total of 38.71 Gb clean data from 6 cDNA libraries were retained (Table S1).

\section{Mapping pair-end reads to the reference genome}

The 'Tuxedo' package HISAT-StringTie [23] was utilized to process the RNAseq data. The reference genome (https://ndownloader.figshare.com/files/16341227) and gene model annotation file (https://ndownloader. figshare.com/files/16341245) were downloaded from Figshare [24].

The RNAseq reads for each sample were mapped to the reference genome using HISAT2, and the output SAM files were sorted and converted to BAM files using SAMtools (version 0.1.19). Then the sorted alignments were assembled into transcripts and the expression levels of all genes and transcripts were estimated using StringTie.

\section{Analysis of the differentially expressed genes (DEGs)}

The expression values were represented by fragments per kilobase transcript per million reads mapped (FPKM), and the differential expression analysis of genes and transcripts across two conditions was performed using the Cuffdiff utility. Foldchange $\geq 2$ and FDR $\leq 0.05$ was set as the threshold to determine the DEGs between the compared samples. The KEGG pathway enrichment analysis of DEGs was conducted by Path_finder software with Q-value $\leq 0.1[19]$.

\section{Integrative targeted metabolomic and transcriptomic profiling analysis}

The data of metabolites profiling were normalized and exported to Simca-P software (12.0, http://www. umetrics.com/simca) employing partial least-squares discriminant analysis (PLS-DA) model. The differentially expressed metabolites were discriminated according to a threshold of variable importance in the projection (VIP) values (VIP $>1$ ) after PLS-DA processing using the previously published protocols [17].

The correlation among lignan biosynthetic genes and lignans was constructed using the Pearson correlation coefficient according to the co-occurrence principle. The correlation network was generated using Cytoscape [17].

\section{qRT-PCR Analysis}

In order to verify the differentially expressed genes (DEGs), the total RNA of 3 individuals of each genotype which were used for the aforementioned metabolite profiling was extracted by Total RNA Kit II (Qiagen). Then, DNaseI treatment, RNA concentration measurement and cDNA synthesis were carried out. According to the RNA-seq data, Primer5 software was used to design primer pairs for randomly selected DEGs (primers were listed in the supplementary Table S2). The housekeeping gene UBIQUITIN1 was used as the reference gene to calculate the relative expression of genes using the comparative Ct method [11].

\section{Results}

Metabolomic alterations in Isatidis Radix following autopolyploidization

In order to assess the impact of polyploidization on the metabolomic shifts, the extracts from Isatidis Radix autotetraploids and their diploid parents were subjected to the targeted metabolic profiling by UPLC-TOF/MS. Totally, 283 annotated metabolites were identified in Isatidis Radix, the roots of I. indigotica.

For the 70 polyploidization-altered metabolites (VIP > 1 and $\left|\log _{2} \mathrm{FC}\right|>1$ ) (Fig. 1), they were mainly enriched in Alkaloids (including quinolines), Phenolic acids, Lignans, and Flavonols (Table S3). Given that lignans and flavonoids are the two major classes of phenylpropanoids in I. indigotica, the obtained results illustrated the polyploidy-inducibility of phenylpropanoids biosynthesis in Isatidis Radix obviously (Fig. 2).

Compared with the diploid parent, the content of Fraxetin-7,8-di-O-glucoside was maintained at a higher level at autotetraploid $\left(\log _{2} \mathrm{FC}=12.8\right)$, whereas 4Methylamyl Glucosinolate was in the reverse trend $\left(\log _{2} \mathrm{FC}=-21.3\right)$. Next, p-Coumaric acid-4-O-glucoside $\left(\log _{2} \mathrm{FC}=11.63\right)$ and 3-Hydroxy-5-Methylphenol-1-OGlucoside $\left(\log _{2} \mathrm{FC}=11.62\right)$ represented the second and the third largest upregulated metabolites following polyploidization, respectively, while 1'-O-(3,4-Dihydroxyphenethyl)-O-caffeoyl-glucoside ( $\left.\log _{2} \mathrm{FC}=-16.6\right)$ and Caffeic acid $\left(\log _{2} \mathrm{FC}=-15.5\right)$ was the second and the third significantly downregulated compounds by polyploidization, respectively (Fig. 3, Table S3).

It is noteworthy that the biosynthesis of Lariciresinol glucosides was enhanced in autopolyploid (Fig. 3, Table S3). Regarding the flavonoid, the accumulation of Phlorizin was enhanced in autotetraploid in comparison to diploid (Table S3). These compounds can be regarded as the indictor components of polyploidization based on metabolomics data. 


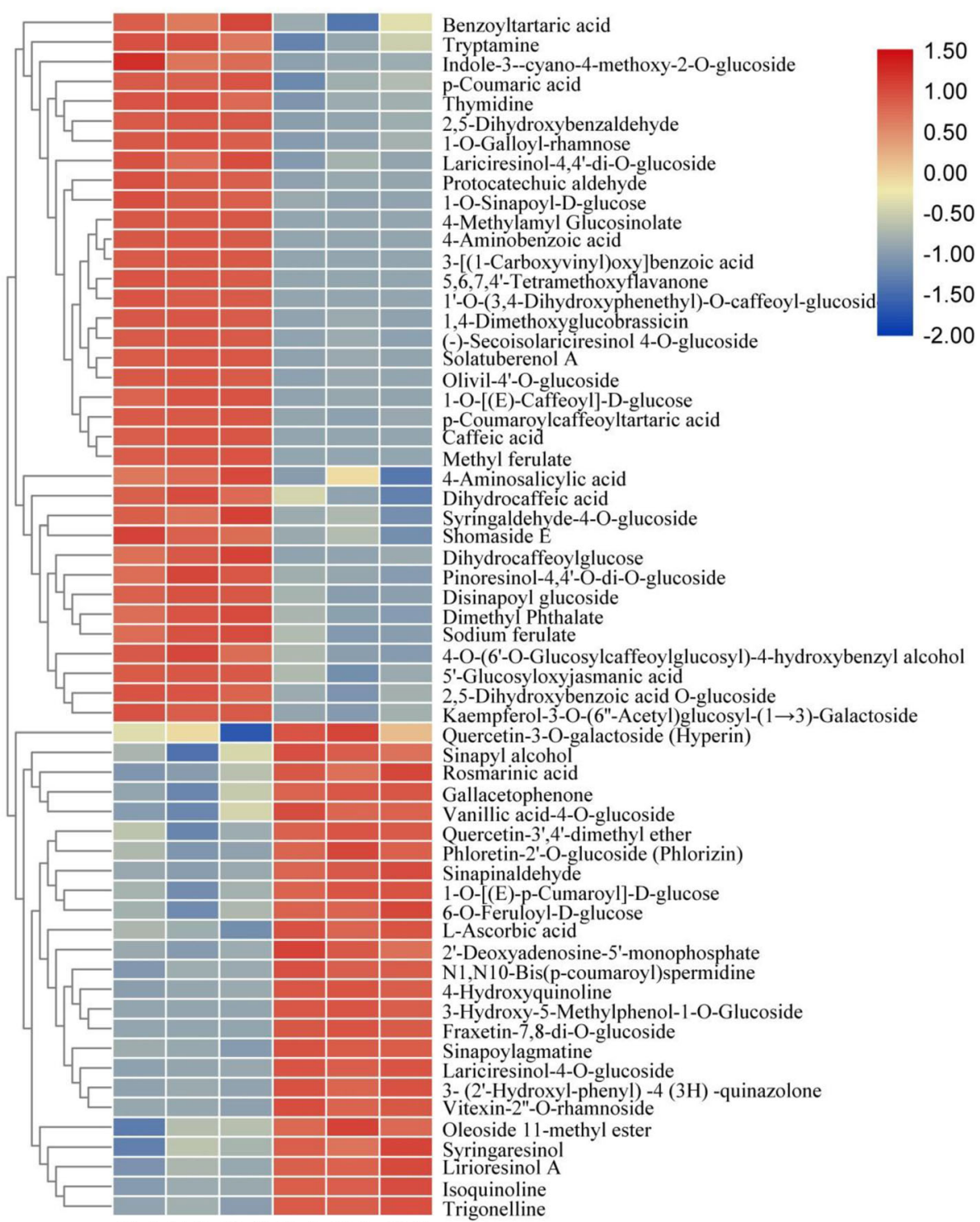

D-1 D-2 D-3 T-1 T-2 T-3

Fig. 1 The polyploidization-altered metabolitesin Isatidis Radix. D and T with triplicate represents for Isatidis Radix diploid and tetraploid, respectively. Those polyploidization-altered metabolites (VIP > 1 and FC > 2) with no structural isomers were shown in the Heatmap

\section{Polyploidization-modulated changes in lignan biosynthesis}

Metabolic analysis revealed that the production of lignans was significantly increased post polyploidization, since Coniferyl alcohol and Lariciresinol glucosides accumulated more in autotetraploid than in diploid. Moreover, Coniferyl aldehyde and the subsequent Coniferyl alcohol, the critical precursor for lignan biosynthesis, were all enhanced in autotetraploid.

However, ferulic acid, the precursor of Coniferyl alcohol as well as Sinapyl alcohol, was less accumulated in autotetraploid than in diploid. Pinoresinol and its derivatives (Pinoresinol-4-O-glucoside, Pinoresinol-4,4'-O-diO-glucoside) displayed less accumulation in autotetraploid than in diploid, as was the case for Secoisolariciresinol glucoside or Lariciresinol-4,4'-di-O-glucoside (also named clemastanin B) (Table S3), which implied that polyploidization-modulated changes in lignan biosynthesis.

\section{Polyploidization-responsive genes in Isatidis Radix}

RNAseq-based transcriptomic profiling was performed to investigate the polyploidization imposed profound impacts on gene expression and the subsequent metabolic pathways in Isatidis Radix. Using a stringent cutoff (Foldchange $>2$ and FDR $\leq 0.05$ ), a total of 2065 differentially expressed genes (DEGs) were identified as being polyploidization-responsive genes, of which 1251 were 


\section{Statistics of KEGG Enrichment}

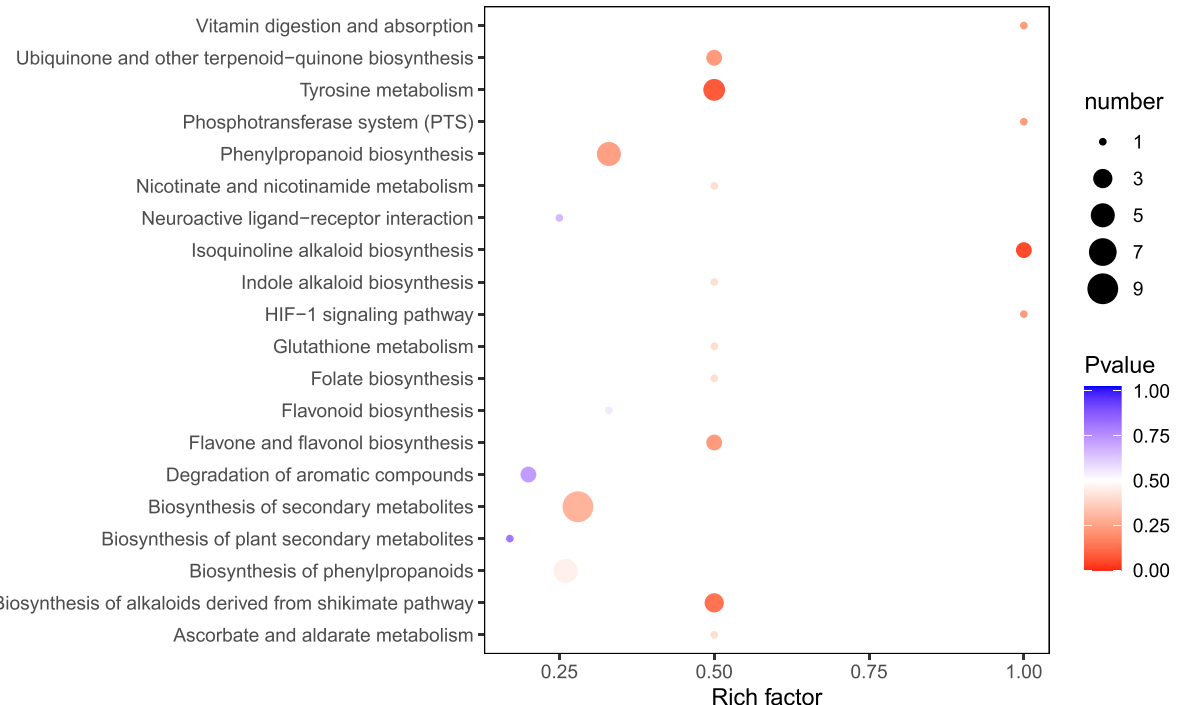

Fig. 2 Polyploidy-inducibility of phenylpropanoids biosynthesis in Isatidis Radix

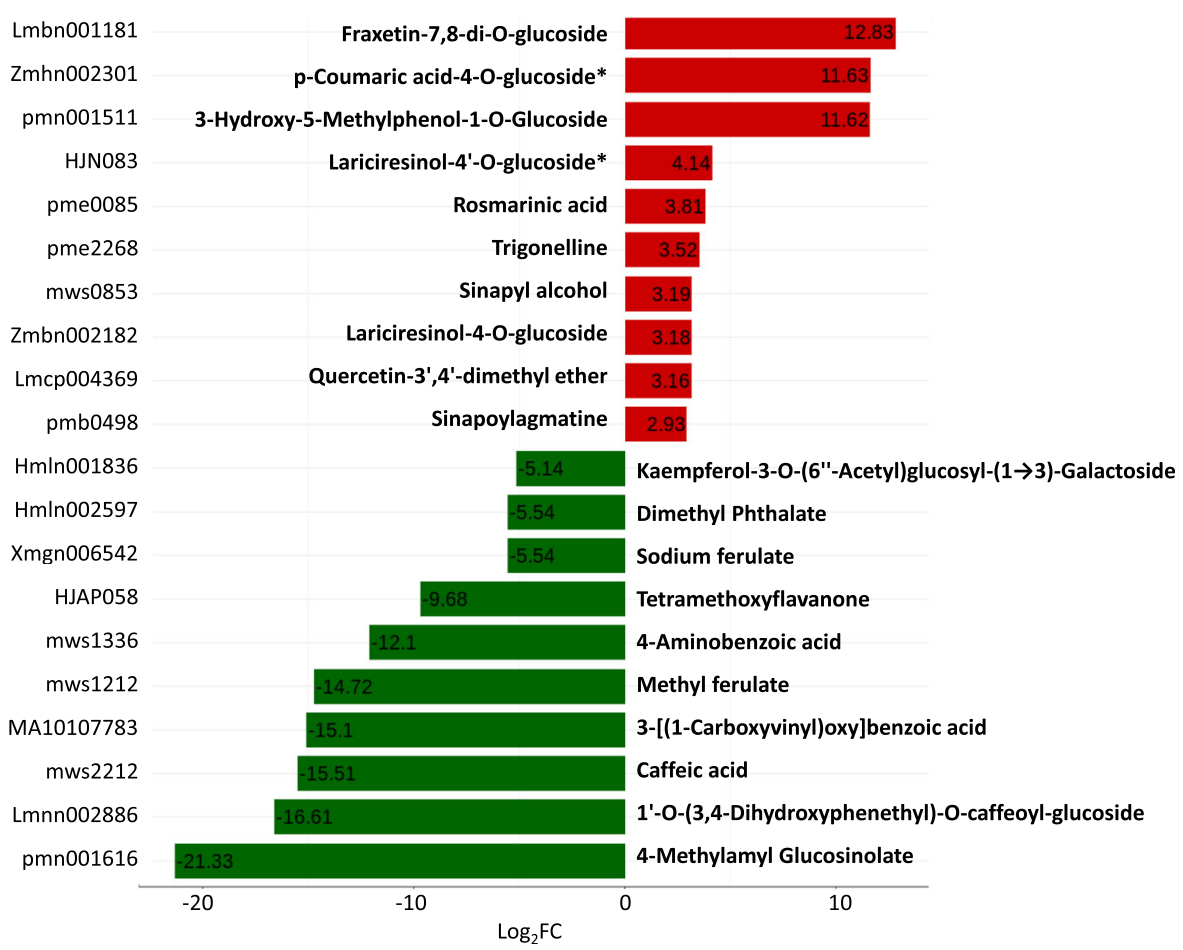

Fig. 3 Top 20 altered metabolites in Isatidis Radix following autopolyploidization. The number in the colored band means log 2 (tetraploid/ diploid). The metabolites with the star signal * mean structural isomer detected 
polyploidy-induced and 814 were polyploidy-repressed in I. indigotica seedlings roots (Table S4). To further evaluate the functions and the biological pathways represented by the DEGs, we compared these genes with that included in the KEGG database [25]. The annotation and classification of root DEGs indicated that the polyploidization-altered root genes were enriched in phenylpropanoid biosynthesis and plant hormone signal transduction (Fig. 4).

To gain insights into the functionality of the 2065 DEGs that are likely to be associated with the process of polyploidization, all of these polyploidy-responsive transcripts were functionally grouped (Table 1). Among DEGs mainly involved in stress response, L-ascorbate peroxidase S, trehalose-phosphate synthase TPS7 and Senescence/dehydration-associated protein were upregulated. Regarding the upregulated DEGs involved in growth and development, Light-regulated protein 1, GIGANTEA, Glycine-rich protein 3 and two HAIKU members were of particular interest. In the kinase and signaling category, three kinase (Receptor-like protein kinase FERONIA, Wall-associated receptor kinase WAK14 and Mitogen-activated protein kinase MPK19) and Gibberellin receptor GID1C were upregulated by polyploidization. Regarding the transporters, Glutathione S-transferase GSTZ1, Sulfate transporter AST12 and two ABC transporter C family members (ABCG14/36) were upregulated by polyploidization (Table 1 ).
Furthermore, 26 polyploidy-altered transcriptional factors (FPKM > 10 in one sample) were identified in Isatidis Radix (Table 2). Among them, 18 transcriptional factors TFs (including bZIP40, NAC29, Myb59) and 8 TFs (including ERF36/70 and NAC41) were upregulated and down-regulated by polyploidization, respectively.

\section{Systematic transcriptomic and metabolomic shift post polyploidization}

To integrate the analysis of polyploidy-responsive metabolome and transcriptome, a canonical correlation analysis using Pearson's correlation coefficient was performed to display the dynamic variation over the polyploidization course. This integrative analysis showed that 1584 DEGs were highly correlated with the 70 polyploidization-altered metabolites, with $|\mathrm{PCC}|>0.917$ (Table S5).

A TF-metabolite correlation network was built that consisted of 15 polyploidy-altered TFs and 67 fluctuated compounds to characterize TFs involved in polyploidyinduced alteration in roots metabolome and transcriptome (Fig. 5, Table S6).

The polyploidy-downregulated metabolites or transcriptional factor genes are marked with background in green circle or oval, respectively. Red lines indicate the positive correlations while blue lines indicate the negative correlation. The polyploidy-altered pattern

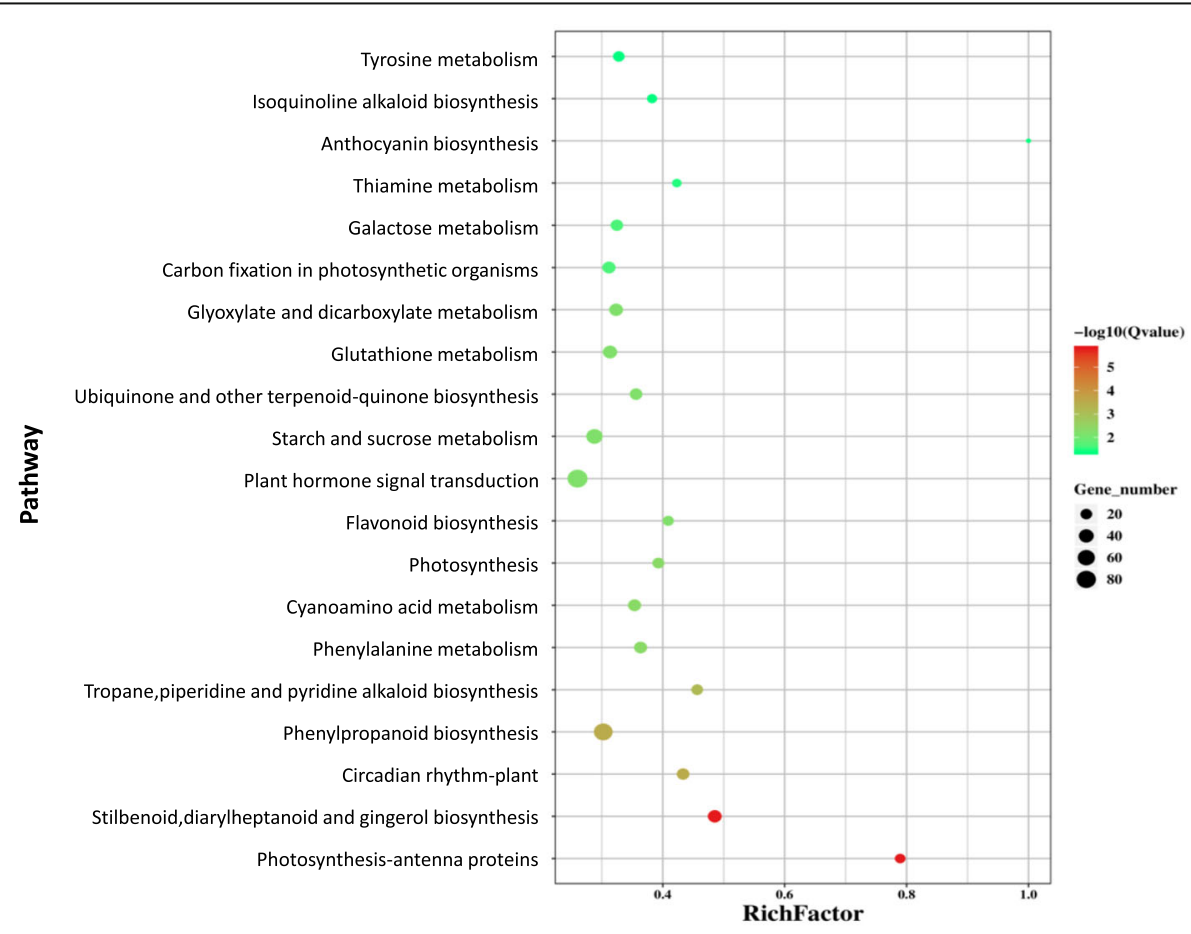

Fig. 4 The KEGG pathway enrichment analysis of polyploidy-altered DEGs in Isatidis Radix 
Table 1 Functional classification of some differentially expressed genes (DEGs) in Isatidis Radix

\begin{tabular}{|c|c|c|}
\hline \multicolumn{3}{|c|}{ Polyploidy-upregulated } \\
\hline Gene ID & $\log _{2} \mathrm{FC}$ & Annotation \\
\hline \multicolumn{3}{|l|}{ Stress } \\
\hline $\operatorname{lin} 06055$ & 2.2 & Temperature-induced lipocalin-1 \\
\hline lin07195 & 6.1 & $\begin{array}{l}\text { L-ascorbate peroxidase S, chloroplastic/ } \\
\text { mitochondrial }\end{array}$ \\
\hline $\operatorname{lin} 08766$ & 1.2 & Trehalose-phosphate synthase TPS7 \\
\hline $\operatorname{lin} 11872$ & 3.9 & Cysteine proteinase $\mathrm{RD} 21 \mathrm{~A}$ \\
\hline $\operatorname{lin} 26982$ & 1.5 & Bifunctional enolase 2/transcriptional activato \\
\hline $\operatorname{lin} 27472$ & 1.8 & Galactinol-sucrose galactosyltransferase 2 \\
\hline $\operatorname{lin} 21449$ & 1.2 & $\begin{array}{l}\text { Senescence/dehydration-associated protein, } \\
\text { chloroplastic }\end{array}$ \\
\hline $\operatorname{lin} 15549$ & 3.8 & Universal stress protein A-like protein \\
\hline
\end{tabular}

\section{Polyploidy-downregulated}

Gene ID $\quad \log _{2}$ FC Annotation

$\operatorname{lin} 16550 \quad-1.7 \quad$ Universal stress protein A-like protein

lin20218 $\quad-1.6 \quad$ Thioredoxin H2

lin26513 -1.2 Gamma carbonic anhydrase CAL2, mitochondrial

lin28989 -2.2 Polyadenylate-binding protein PABP-2

lin02876 -3.6 Jacalin-related lectin 35

lin24588 -1.2 Glutathione S-transferase DHAR2

\section{Growth and development}

\begin{tabular}{|c|c|c|}
\hline $\operatorname{lin} 01550$ & 5.7 & Light-regulated protein 1, chloroplastic \\
\hline $\operatorname{lin} 10290$ & 2.2 & Protein GIGANTEA \\
\hline $\operatorname{lin} 15495$ & 2.5 & $\begin{array}{l}\text { Mediator of RNA polymerase II transcription } \\
\text { subunit } 37 \mathrm{c}\end{array}$ \\
\hline $\operatorname{lin} 02027$ & 1.7 & HAIKU1NQ14 \\
\hline $\operatorname{lin} 13953$ & 3.2 & Glycine-rich protein 3 \\
\hline $\operatorname{lin} 00462$ & 2.9 & BTB/POZ and TAZ domain-containing protein 1 \\
\hline $\operatorname{lin} 04656$ & 3.7 & MADS-box protein FLOWERING LOCUS C \\
\hline $\operatorname{lin} 05649$ & 2.0 & Nucleolin 1 \\
\hline $\operatorname{lin} 08418$ & 4.8 & E3 ubiquitin ligase SUD1 \\
\hline $\operatorname{lin} 09123$ & 1.8 & Receptor-like protein kinase HAIKU2 \\
\hline $\operatorname{lin} 09717$ & 2.5 & $\begin{array}{l}\text { Glyceraldehyde-3-phosphate dehydrogenase } \\
\text { GAPCP2, chloroplastic }\end{array}$ \\
\hline & & $\mathrm{c}$ \\
\hline
\end{tabular}

$\begin{array}{lll}\operatorname{lin} 18645 & -1.6 & \text { Arogenate dehydratase PDT1, chloroplastic } \\ \text { lin05822 } & -1.3 & \text { Adenylate kinase isoenzyme 6 homolog } \\ \operatorname{lin} 10029 & -3.7 & \text { Auxin-responsive protein SAUR50 } \\ \text { lin11047 } & -1.7 & \text { Rop guanine nucleotide exchange factor 14 } \\ \operatorname{lin} 12932 & -3.2 & \text { Profilin-1 } \\ \operatorname{lin} 23516 & -1.2 & \text { Rac-like GTP-binding protein ARAC3 } \\ \operatorname{lin} 00261 & -1.2 & \text { Agamous-like MADS-box protein AGL31 } \\ \operatorname{lin} 07151 & -2.7 & \text { DNA (cytosine-5)-methyltransferase 1 } \\ \operatorname{lin} 12525 & -11.7 & \text { Uracil phosphoribosyltransferase, chloroplastic } \\ \operatorname{lin} 17933 & -1.1 & \text { Pyruvate dehydrogenase E1 component subunit } \\ & & \text { beta-3, chloroplastic } \\ \operatorname{lin} 19209 & -1.3 & \text { UDP-sugar pyrophosphorylase } \\ \operatorname{lin} 25514 & -1.6 & \text { Zinc finger protein 6 } \\ \operatorname{lin} 27501 & -1.3 & \text { SUMO-conjugating enzyme SCE1 } \\ \operatorname{lin} 29572 & -1.9 & \text { Adenosylhomocysteinase 1 }\end{array}$

\section{Kinase and signaling}

$\begin{array}{lll}\text { lin26824 } & 1.2 & \text { Receptor-like protein kinase FERONIA } \\ \text { lin19551 } & 1.7 & \text { Wall-associated receptor kinase, WAK14 } \\ \text { lin24110 } & 6.0 & \text { Mitogen-activated protein kinase, MPK19 } \\ \text { lin27283 } & 1.2 & \text { receptor-like protein kinase } \\ \text { lin29680 } & 4.3 & \text { LRR receptor-like kinase } \\ \text { lin01264 } & 1.0 & \text { LRR receptor-like kinase } \\ \text { lin00785 } & 2.2 & \text { Remorin } \\ \text { lin01090 } & 1.8 & \text { Gibberellin receptor GID1C } \\ \text { lin07657 } & 3.1 & \text { Copper amine oxidase }\end{array}$

$\begin{array}{lrl}\text { lin17733 } & -2.2 & \text { LRR receptor-like protein kinase PXC1 } \\ \text { lin01347 } & -1.1 & \text { Histidine-containing phosphotransfer protein 2 } \\ \text { lin03883 } & -1.6 & \text { Phosphoglucan, water dikinase, chloroplastic } \\ \text { lin10356 } & -7.3 & \text { Hexokinase-like 1 protein } \\ \text { lin28764/ } & -1.6 & \text { RPM1-interacting protein 4 } \\ \text { lin30061 } & & \\ \text { lin22433 } & -5.3 & \text { F-box protein GID2 } \\ \text { lin29325 } & -2.2 & \text { Auxin-responsive protein IAA7 } \\ \text { lin05103 } & -1.7 & \text { Gibberellin-regulated protein 4 } \\ \text { lin06703/ } & -1.1 & \text { Small acidic protein 1 } \\ \text { lin21095 } & & \\ & & \\ \text { lin22173 } & -1.7 & \text { Calcium permeable stress-gated cation channel, } \\ \text { lin11860 } & -1.3 & \text { ABC transporter A family, ABCA4 } \\ \text { lin04029 } & -1.3 & \text { Membrane magnesium transporter }\end{array}$

\section{Transporter}

$\begin{array}{lll}\text { lin01771 } & 2.7 & \text { Glutathione S-transferase, GSTZ1 } \\ \text { lin04670 } & 1.6 & \text { Ras-related protein, RABH1e } \\ \operatorname{lin} 26854 & 2.3 & \text { Sulfate transporter, AST12 }\end{array}$


Table 1 Functional classification of some differentially expressed genes (DEGs) in Isatidis Radix (Continued)

\begin{tabular}{lllllll}
\hline \multicolumn{2}{l}{ Polyploidy-upregulated } & & \multicolumn{3}{l}{ Polyploidy-downregulated } \\
\cline { 1 - 2 } \cline { 5 - 6 } Gene ID & Log $_{2}$ FC & Annotation & & Gene ID & Log $_{2}$ FC & Annotation \\
\hline $\operatorname{lin} 10902$ & 1.8 & CSC1-like protein, ERD4 & $\operatorname{lin} 28450$ & -0.8 & Mitochondrial phosphate carrier protein, MPT3 \\
$\operatorname{lin} 01411$ & 2.2 & AAA-ATPase & $\operatorname{lin} 22985$ & -5.9 & Venom phosphodiesterase \\
$\operatorname{lin} 27921 /$ & 1.6 & ABC transporter C family, ABCG14/36 & & & \\
$\operatorname{lin} 23995$ & 1.1 & Amino acid transporter, AVT3C & & & \\
$\operatorname{lin} 23984$ & 1.1 & & & & \\
\hline
\end{tabular}

Log 2 FC means $\log _{2}$ (FPKM-tetraploid/FPKM-diploid). FPKM means the fragments per kilobase transcript per million reads mapped by RNAseq analysis

and annotation of metabolites or genes are given in Table S6.

Among the 15 polyploidy-altered TFs, bHLH44/63/129, ERF70, ASIL2, NF-YC-1 and HsfB2b were highly connected to the fluctuated compounds. It is also intriguing to note that the polyploidy-induced NAC54 was positively correlated with polyploidization-enhanced two metabolites (Sinapyl alcohol and Quercetin-3',4'-dimethyl ether).
For the polyploidization-enhanced metabolites, three types of Quinolines, Gallacetophenone, Sinapyl alcohol, $p$-Coumaric acid-4-o-glucoside, Vanillic acid-4-o-glucoside, Rosmarinic acid, Lirioresinol A, Syringaresinol, Lariciresinol-4-o-glucoside, Fraxetin-7,8-di-o-glucoside, Vitexin-2"-o-rhamnoside were all positively correlated with 4 polyploidy-induced TFs (HsfB2b, ASIL2, bHLH129, bHLH63).

Table 2 Polyploidy-altered transcriptional factors in Isatidis Radix

\begin{tabular}{|c|c|c|c|c|c|c|c|c|c|}
\hline Name & Diploid & Tetraploid & $\log _{2} F C$ & ID & Chromosome & Start & End & Strand & Annotation \\
\hline ARF14 & 7.2 & 62.6 & 3.2 & $\operatorname{lin} 01634$ & Lachesis_group0 & $8,536,701$ & $8,537,541$ & - & AP2/ERF and B3 domain \\
\hline RAV2 & 2.1 & 13.4 & 2.8 & $\operatorname{lin} 25260$ & Lachesis_group6 & $7,129,978$ & $7,131,110$ & - & AP2/ERF and B3 domain \\
\hline bZIP40 & 29.1 & 61.9 & 1.1 & $\operatorname{lin} 02491$ & Lachesis_group0 & $15,255,544$ & $15,256,922$ & + & bZIP transcription factor \\
\hline CDC5 & 8.7 & 24.3 & 1.5 & $\operatorname{lin} 13523$ & Lachesis_group3 & $7,021,761$ & $7,028,721$ & - & Cell division cycle 5-like \\
\hline ERF36 & 90.0 & 19.1 & -2.2 & $\operatorname{lin} 15129$ & Lachesis_group3 & $27,555,240$ & $27,555,962$ & - & Ethylene-responsive factor \\
\hline ERF55 & 3.2 & 16.5 & 2.4 & $\operatorname{lin} 11510$ & Lachesis_group2 & $20,968,841$ & $20,969,965$ & - & Ethylene-responsive factor \\
\hline ERF70 & 166.1 & 56.9 & -1.5 & $\operatorname{lin} 24996$ & Lachesis_group6 & $5,265,430$ & $5,265,968$ & - & Ethylene-responsive factor \\
\hline HsfAla & 10.9 & 2.4 & -2.2 & $\operatorname{lin} 10321$ & Lachesis_group2 & $10,028,288$ & $10,028,973$ & + & Heat stress transcription factor \\
\hline HsfB2b & 4.8 & 10.2 & 1.1 & $\operatorname{lin} 07593$ & Lachesis_group1 & $30,892,038$ & $30,894,605$ & + & Heat stress transcription factor \\
\hline REV & 3.5 & 22.5 & 2.8 & $\operatorname{lin} 02879$ & Lachesis_group0 & $24,168,399$ & $24,168,936$ & + & Homeobox-leucine zipper \\
\hline NAC41 & 76.6 & 8.8 & -3.2 & $\operatorname{lin} 18045$ & Lachesis_group4 & $8,178,123$ & $8,179,515$ & - & NAC domain-containing \\
\hline NAC29 & 1.9 & 169.4 & 6.5 & $\operatorname{lin} 25189$ & Lachesis_group6 & $6,708,153$ & $6,709,151$ & - & NAC domain-containing \\
\hline NAC47 & 4.5 & 16.4 & 1.9 & $\operatorname{lin} 16287$ & Lachesis_group3 & $33,189,224$ & $33,191,152$ & + & NAC domain-containing \\
\hline NAC54 & 5.7 & 21.9 & 2.0 & $\operatorname{lin} 19433$ & Lachesis_group4 & $29,656,612$ & $29,657,225$ & + & NAC domain-containing \\
\hline$N F-Y C-1$ & 16.9 & 4.2 & -2.0 & $\operatorname{lin} 08913$ & Lachesis_group2 & $2,970,892$ & $2,972,650$ & - & Nuclear transcription factor \\
\hline bHLH44 & 11.2 & 0.1 & -6.9 & $\operatorname{lin} 09913$ & Lachesis_group2 & $7,785,960$ & $7,787,812$ & + & Basic helix-loop-helix protein \\
\hline bHLH129 & 5.4 & 14.0 & 1.4 & $\operatorname{lin} 17039$ & Lachesis_group4 & $2,495,161$ & $2,499,719$ & + & Basic helix-loop-helix protein \\
\hline bHLH130 & 2.0 & 23.3 & 3.6 & $\operatorname{lin} 17124$ & Lachesis_group4 & $2,983,700$ & $2,986,470$ & + & Basic helix-loop-helix protein \\
\hline bHLH63 & 7.0 & 14.7 & 1.1 & $\operatorname{lin} 23473$ & Lachesis_group5 & $35,187,500$ & $35,189,238$ & + & Basic helix-loop-helix protein \\
\hline GTE4 & 21.1 & 76.8 & 1.9 & $\operatorname{lin} 08753$ & Lachesis_group2 & $2,302,922$ & $2,306,873$ & + & Transcription factor GTE4 \\
\hline Myb51 & 6.5 & 31.6 & 2.3 & $\operatorname{lin} 09927$ & Lachesis_group2 & $7,849,190$ & $7,851,367$ & + & MYB Transcription factor \\
\hline Myb59 & 19.5 & 317.8 & 4.0 & $\operatorname{lin} 05872$ & Lachesis_group1 & $10,463,654$ & $10,464,989$ & + & MYB Transcription factor \\
\hline ASIL2 & 13.6 & 29.5 & 1.2 & $\operatorname{lin} 14214$ & Lachesis_group3 & $21,584,634$ & $21,585,940$ & - & Trihelix transcription factor \\
\hline ASR3 & 14.7 & 3.5 & -2.0 & $\operatorname{lin} 23152$ & Lachesis_group5 & $33,490,036$ & $33,491,219$ & - & Arabidopsis SH4-Related3 \\
\hline WRKY16 & 4.3 & 18.5 & 2.2 & $\operatorname{lin} 21936$ & Lachesis_group5 & $26,229,642$ & $26,232,137$ & + & WRKY transcription factor \\
\hline WRKY19 & 13.1 & 0.8 & -3.9 & $\operatorname{lin} 00005$ & Lachesis_group0 & 36,353 & 38,261 & - & WRKY transcription factor \\
\hline
\end{tabular}

The number in the column of Diploid and Tetraploid is the FPKM (fragments per kilobase transcript per million reads mapped) value by RNAseq analysis, and $\log _{2}$ FC means $\log _{2}$ (FPKM-tetraploid/FPKM-diploid) 


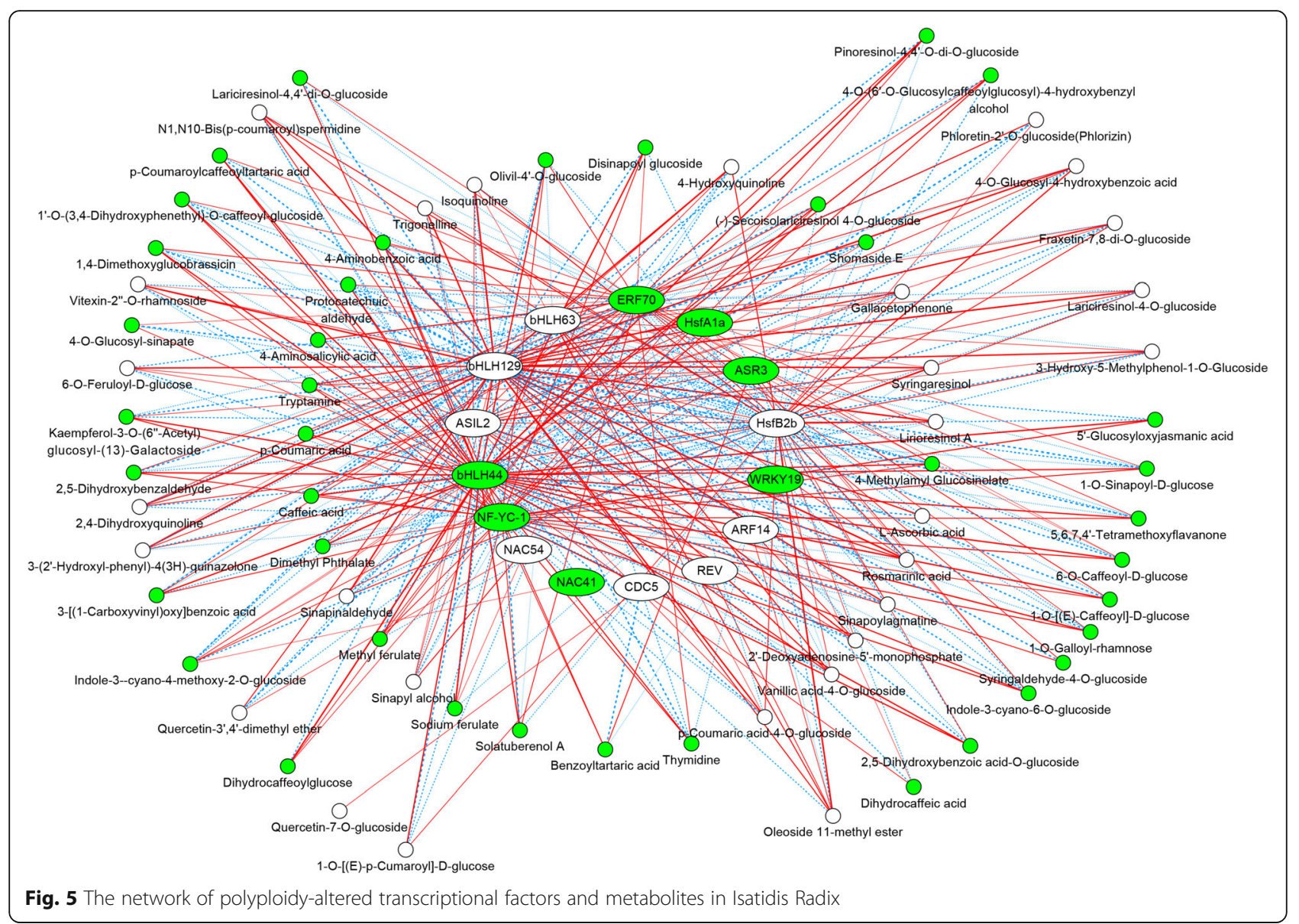

However, the aforementioned metabolites together with Phloretin-2'-O-glucoside (Phlorizin) and Quercetin3',4'-dimethyl ether were all negatively correlated with 3 polyploidy-suppressed TFs (NF-YC-1, bHLH44 and ERF70) except Syringaresinol negatively with NAC41. Moreover, bHLH129 was positively correlated with LAscorbic acid specifically, while it was connected to Phloretin-2'-O-glucoside (Phlorizin) and Quercetin-3',4'dimethyl ether together with other TFs. bHLH44 and ERF70 aforementioned were all negatively correlated with L-Ascorbic acid (Fig. 5, Table S6).

\section{Integrated metabolomic and transcriptomic analysis of lignan metabolism modulated by polyploidization}

To have a systematic view on the polyploidy-responsive variation of lignan biosynthesis, the transcripts involved in the general phenylpropanoid pathway, lignan biosynthesis and the corresponding metabolites were subjected to construct lignan biosynthesis pathway.

Several key metabolites, involved in general phenylpropanoid pathway (e.g. Coniferyl aldehyde and Coniferyl alcohol) [25] and lignan compound (e.g. Lariciresinol glucoside), were markedly increased post polyploidization. Moreover, various catalytic genes (e.g. $\mathrm{C} 4 \mathrm{H}, 4 \mathrm{CL}$,
COMT and $\mathrm{F} 5 \mathrm{H}$ ) showed similar up-regulated patterns in correspondence with the increased metabolites (Fig. 6, Table S3, Table S4), suggesting lignan biosynthesis pathway modulated by polyploidization with transcriptomic and metabolomics evidence.

PAL, phenylalanine ammonia-lyase; UGT, UDP-sugardependent glycosyltransferase; $\mathrm{C} 3 \mathrm{H}, \quad p$-coumarate 3hydroxylase, 4CL, 4-(hydroxy) cinnamoyl CoA ligase; $\mathrm{CCR}$, cinnamoyl-CoA reductase; $\mathrm{F} 5 \mathrm{H}$, ferulate-5-hydroxylase; CAD, cinnamyl alcohol dehydrogenase; DIR, dirgent protein; PLR, pinoresinol/lariciresinol reductase; SIRD, secoisolariciresinol dehydrogenase. Metabolites or catalytic genes up-regulated post polyploidization were marked in red, and those down-regulated post polyploidization were in blue. The polyploidy-altered metabolites were shown in box, while the polyploidy-altered genes were placed adjacent to the arrows. Metabolites or genes in black means not significantly regulated by polyploidization, and the polyploidy-altered pattern and annotation information of metabolites or genes are given in supplementary Table S3 and Table S4, respectively.

In the polyploidy-altered TFs-lignans network, there were 10 polyploidy-altered TFs and 17 fluctuated phenylpropanoid pathway compounds, which indicated the 


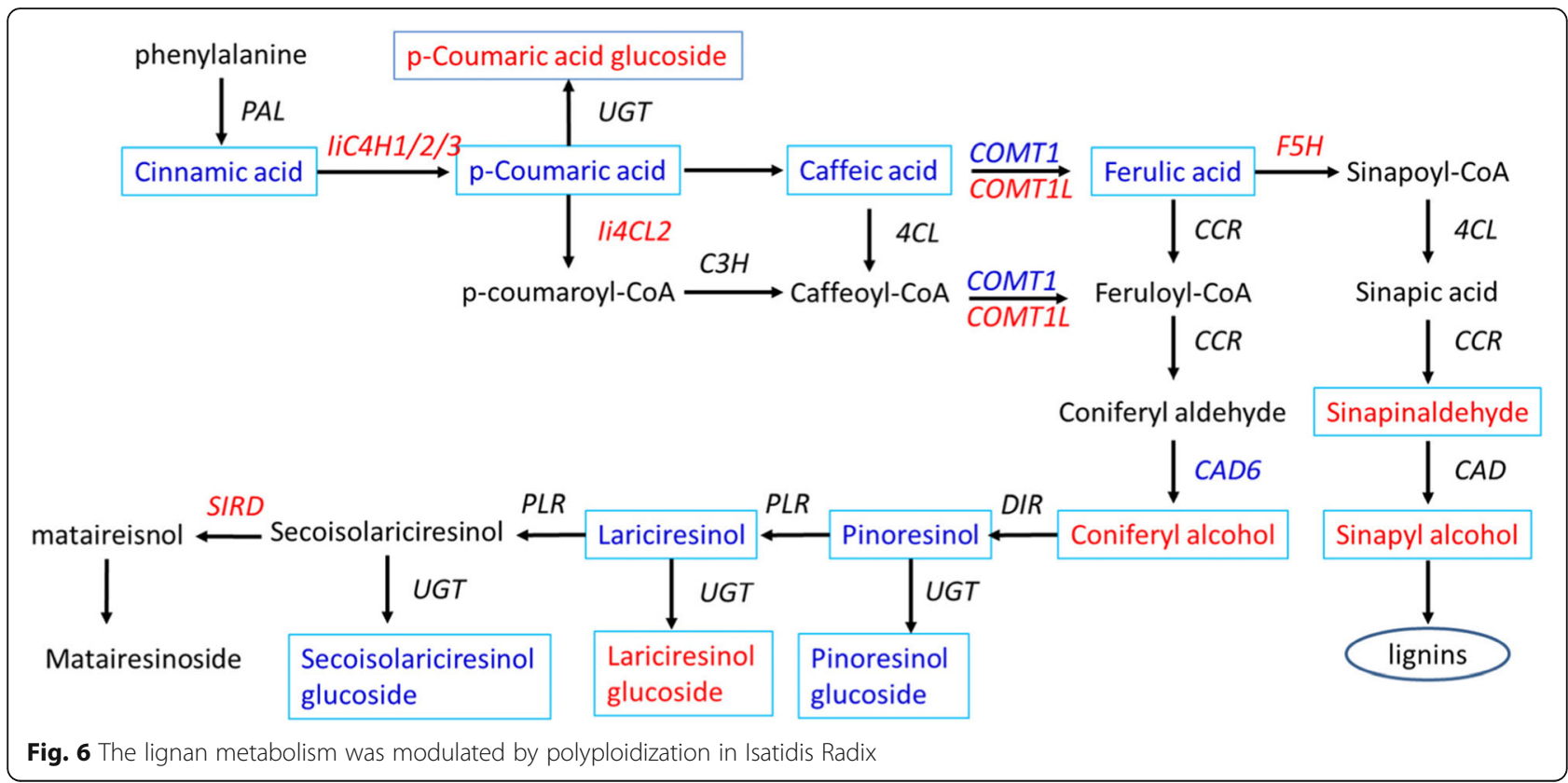

transcriptomic and metabolic shifts in lignan metabolism as a result of polyploidization-mediated transcriptional regulation (Fig. 7, Table S7).

Among the 10 polyploidy-altered transcriptional factors, polyploidy-inhibited bHLH44 and polyploidyinduced bHLH129 were highly correlated with all the fluctuated phenylpropanoid pathway compounds (7 up and 10 down) except Syringaresinol, but with the reverse trend.

The polyploidization-enhanced Lariciresinol-4-O-glucoside and polyploidization-reduced (-)-Secoisolariciresinol 4-O-glucoside were correlated with 4 polyploidyinduced TFs (HsfB2b, ASIL2, bHLH129, bHLH63) and 3 polyploidy-suppressed TFs (NF-YC-1, bHLH44 and

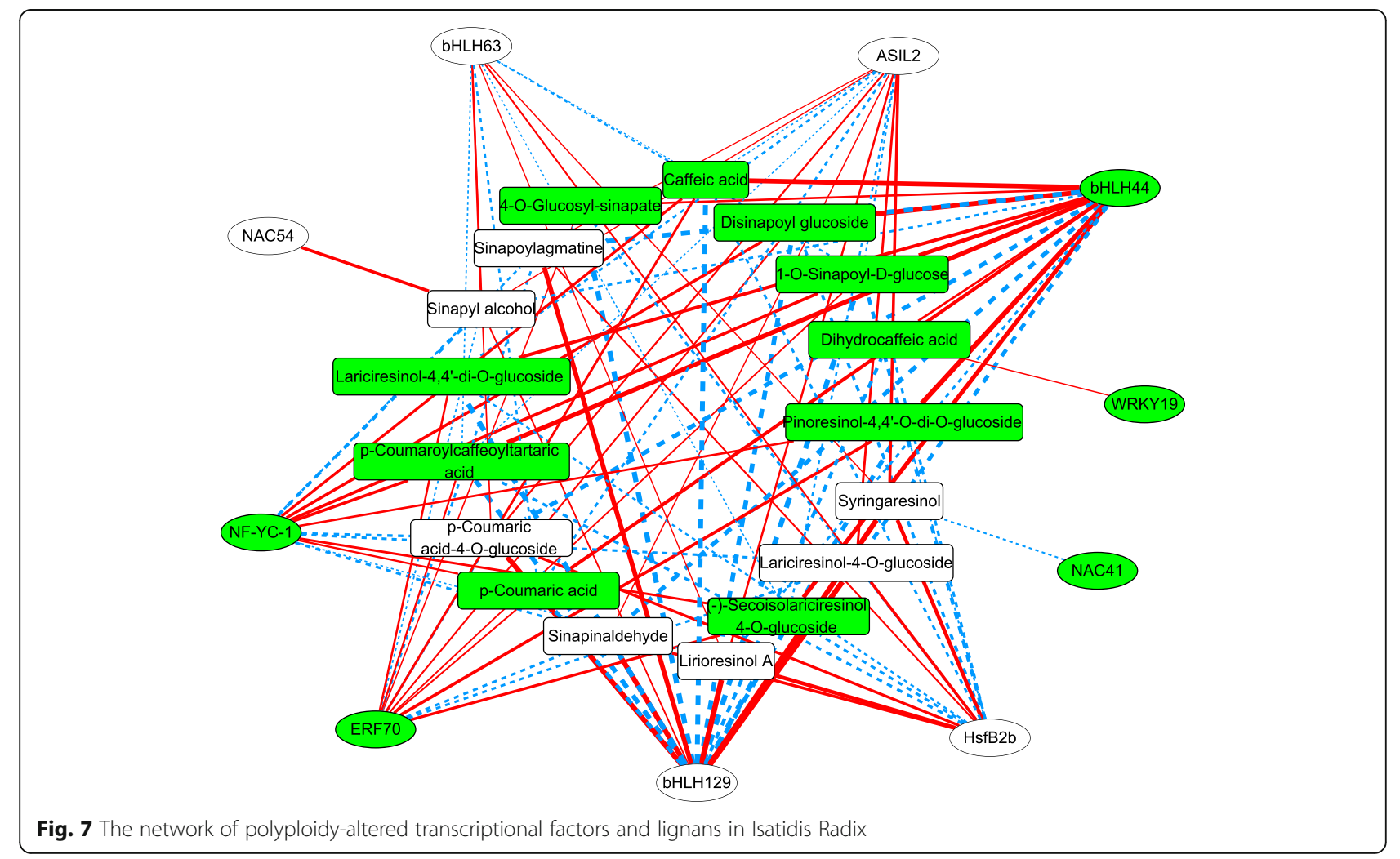


ERF70) with reverse trend, so was the case for the polyploidization-reduced Lariciresinol-4,4'-di-O-glucoside correlated with the aforementioned TFs except bHLH63 or NF-YC-1 (Fig. 7, Table S7).

The polyploidy-downregulated metabolites or genes are marked with background in green box or oval, respectively. Red lines indicate the positive correlations while blue lines indicate the negative correlation. The polyploidy-altered pattern and annotation of metabolites or genes are given in supplementary Table S7.

\section{Discussion}

Polyploidization contributed to the 'gigas effect' and high content of active compounds in I. indigotica autotetraploid roots

Polyploidization, also known as whole-genome duplication (WGD), results in the "gigas effect" that includes increased cell size, enlarged vegetative or reproductive organs and prolonged vegetative growth $[11,15,26]$. Compared to their diploid progenitors, the autotetraploid I. indigotica plants exhibit bigger robustness and larger leaves with deeper color, which was in accordance with the "gigas effect".

Among DEGs mainly involved in stress response, Lascorbate peroxidase $S$, trehalose-phosphate synthase (TPS7) and senescence/dehydration-associated protein were upregulated by polyploidization (Table 1). In Arabidopsis, TPS1 catalyzes the synthesis of the sucrosesignaling metabolite trehalose 6-phosphate which acts as a potent regulator of post-embryonic growth and development [27]. Moreover, rice OsTPS1 may improve the abiotic stress tolerance by increasing the accumulation of trehalose and proline, and modulating the expression of stress-related genes [28]. Regarding the senescence/ dehydration-associated protein, Arabidopsis ERD7 and its homologs play essential roles in plant stress responses and development and are associated with modification of membrane lipid composition [29]. Therefore, the role of these polyploidy-enhanced genes in 'gigas effect' and stress tolerance of autotetraploid needs further establishment.

Regarding the kinase genes upregulated by polyploidization, the receptor-like protein kinase FERONIA was of particular interest (Table 1). In Arabidopsis, the couple of extracellular peptide RAPID ALKALINIZATION FACTOR1 (RALF1) and FERONIA (FER) acted as a central hub between the cell surface and downstream signaling events, and the RALF-FER pathway functioned as an essential regulator of plant stress responses [30]. Furthermore, the RALF1-FER-GRP7 module provided a paradigm for regulatory mechanisms of RNA splicing to regulate plant fitness and flowering time [30, 31]. Glycine-rich proteins (GRPs) were demonstrated to participate in cold stress responses, plant defense, cell elongation and fertility. Moreover, rice glycine-rich protein OsDG2 plays important roles in chloroplast development during early seedling stage [32]. In this study, glycine-rich protein (Iin13953) was one of the polyploidy-upregulated DEGs which were involved in growth and development (Table 1). Hence, it is interesting to investigate the contribution of polyploidy-induced FER together with glycine-rich protein Iin13953 to the modulation of cell growth and stress responses.

Arabidopsis root hair defective 6-like 4 (RSL4), a bHLH transcription factor, triggers the expression of hundreds of root hair genes which promote ectopic root hair growth, and the autocrine regulation of root hair size by the RALF-FERONIA-RSL4 signaling pathway has been revealed [33]. In this study, polyploidy-induced bHLH129 and bHLH63 together with FER were identified (Tables 1 and 2), but whether they acted as central hubs orchestrating complex intracellular and extracellular signals required further elucidation.

One of the ideal expectations for the medicinal autopolyploid was that the organ giantism was accompanied by the higher content of some chemical compositions, especially the active compounds $[8,13-16,19]$. I. indigotica, like I. tinctoria of the Brassicaceae family, represents a valuable source of bioactive compounds such as alkaloids, phenolic compounds, phenylpropanoids and terpenoids $[3,19]$. In this study, the content of active compounds in the roots of autotetraploid I. indigotica was higher than that in diploid roots, and some new compounds including Phlorizin, Tannins and Solatuberenol A were also isolated in Isatidis Radix (Table S3). Moreover, Isatidis Radix autotetraploid accumulated more Lariciresinol glucosides than the diploid counterparts, consistent with previous report [17]. Known indolic alkaloids called Indirubin and indicant (iso) which were reported in the dried I. tinctoria leaves [2,3] were also identified to be polyploidy-upregulated metabolite in this study (Table S3). These collectively implied the potentiality of enhancing active compounds accumulation through polyploidization.

The penetration-resistance gene PEN3/ABCG36/PDR8 and PDR12 function redundantly to mediate the secretion of camalexin, and they have multiple functions in Arabidopsis immunity via transport of distinct $\operatorname{Trp}$ metabolic products [34]. PEN2 encodes a myrosinase that catalyzes the degradation of indole glucosinolates, and the catalyzed products of PEN2 are postulated to be transported to the apoplast by PEN3. Moreover, the indole compound 4-methoxyindole-3-methanol that is a substrate for PEN3 stimulates bacterial flg22-induced callose deposition [35]. In this study, one myrosinase (Iin26136) and two myrosinase-binding protein genes were induced by polyploidization (Table S4). Nonetheless, whether the coordinated function mechanism of 
PEN2-PEN3 play its role in synthesis and export of the active metabolites (including but not limited to indole compounds) in Isatidis Radix merits further investigation.

\section{Gene-lignan pathway network analysis highlighted polyploidy-responsive key functional genes and regulators}

Phenylpropanoid is the major group of secondary metabolites, which metabolism generate diverse metabolites including lignans and flavonoids, and lignans are identified to be the pharmacologically active compounds [7], therefore the correlations between the identified DEGs and phenylpropanoid pathway compounds were inferred based on the co-occurrence principle between the transcript and metabolite levels. Several key metabolites involved in general phenylpropanoid pathway (e.g. coniferyl aldehyde and coniferyl alcohol) and lignan compound (e.g. Lariciresinol glucoside) were markedly increased post polyploidization. Moreover, various catalytic genes (e.g. $\mathrm{C} 4 \mathrm{H}, 4 \mathrm{CL}, \mathrm{COMT}$ and $\mathrm{F} 5 \mathrm{H}$ ) showed similar up-regulated patterns in correspondence with the increased metabolites (Fig. 6), suggesting that transcriptomic and metabolomic profile of lignan biosynthesis pathway was modulated by polyploidization.

Transcriptional factors were predicted to act as key regulators of lignan synthesis in I. indigotica. Among the 10 polyploidy-altered TFs, polyploidy-inhibited bHLH44 and polyploidy-induced bHLH129 were highly correlated with all the fluctuated phenylpropanoid pathway compounds (7 up and 10 down) except Syringaresinol, but with the reverse trend (Fig. 7). It was also intriguing to note that the polyploidy-induced NAC54 was positively correlated with polyploidization-enhanced Sinapyl alcohol (Fig. 7). Further studies of the regulatory mechanism of polyploidy-induced bHLH129 and NAC54 may provide fruitful means to reveal the beneath mechanism for polyploidy vigor and lignan biosynthesis of I. indigotica.

It was reported that IiWRKY34 significantly contributed to the polyploidy vigor of I. indigotica, and IiWRKY34 positively contributed to the yield, lignan biosynthesis and stress tolerance in I indigotica hairy roots, however, this key regulator was not identified here using the genuine roots namely Isatidis Radix. One possible explanation is that the expression pattern of genes in the induced hairy roots of tetraploid I. indigotica greatly differed from that in its original root [17].

Not surprisingly, different TFs may play distinct roles in lignan biosynthesis and allow the autotetraploid roots to prioritize toward a more efficient lignan biosynthesis. Therefore, whether these highlighted TFs regulate DEGs for lignan biosynthesis is the most important issue to elucidate the genuine regulators of lignan biosynthesis in Isatidis Radix.

\section{Supplementary Information}

The online version contains supplementary material available at https://doi. org/10.1186/s12864-021-07980-w.

Additional file 1: Table S1. Quality statistics of the filtered RNAseq reads.

Additional file 2: Table S2.qRT-PCR primers of Isatidis Radix genes. Additional file 3:Table S3. Metabolomic profiling of Isatidis Radix. Additional file 4: Table S4. Polyploidy-responsive genes in Isatidis Radix.

Additional file 5: Table S5. Correlation analysis of polyploidyresponsive genes and metabolites in Isatidis Radix.

Additional file 6: Table S6. Correlation analysis of transcriptional factors and metabolites in Isatidis Radix.

Additional file 7: Table S7. I. indigotica transcriptional factors acted as key regulators of lignan synthesis in Isatidis Radix.

Additional file 8: Figure S1. The morphological characterization of I. indigotica autotetraploid seedling and its diploid progenitor. A The I. indigotica seedling of autotetraploid (4x) and its diploid (2x). B

Chromosomes of I. indigotica autotetraploid and diploid root tips. C The comparison of stomata between autotetraploid and diploid leaf. D Isatidis Radix autotetraploid and diploid.

\section{Acknowledgements}

We thank Jiangsu Germplasm Repository Center for providing I. indigotica Fort. $(2 n=2 x=14)$ as the donor for polyploidization, Wuhan Frasergen Bioinformatics Co.,Ltd. for technical assistance in RNA sequencing and MetWare Biotechnology Co.,Ltd. for targeted metabolites analysis.

\section{Authors' contributions}

$Z X, M T$ and $Z Z$ conceived and designed the research. ZZ, YJ, JW, JZ and YL performed the experiments. MT, YZ, ZZ, SZ collated the data. MT, ZZ and $Y Z$ wrote and revised the manuscript. All authors read and agreed to the published version of the manuscript.

Funding

This study was supported by China Agriculture Research System (CARS-21).

\section{Availability of data and materials}

The datasets supporting the conclusions of this article are included within the article and its additional files.

\section{Declarations}

Ethics approval and consent to participate

The I. indigotica seeds used in this study were kindly provided by Jiangsu Germplasm Repository Center, and the samples collected from I. indigotica seedlings were for plant research use only. The use of I. indigotica seeds in this study is complied with the relevant institutional, national, and international guidelines and legislation.

Consent for publication

Not applicable.

\section{Competing interests}

The authors declare no conflict of interest.

\section{Author details}

${ }^{1}$ College of Horticulture, Nanjing Agricultural University, 210095 Nanjing, China. ${ }^{2}$ College of Life Sciences, Nanjing Agricultural University, 210095 Nanjing, China. 
Received: 10 May 2021 Accepted: 25 August 2021

Published online: 17 September 2021

\section{References}

1. Zhang ZJ, Morris-Natschke SL, Cheng YY, Lee KH, Li RT. Development of anti-influenza agents from natural products. Med Res Rev. 2020; 40: 22902338 .

2. Nguyen TK, Marcelo P, Gontier E, Dauwe R. Metabolic markers for the yield of lipophilic indole alkaloids in dried woad leaves (Isatis tinctoria L.). Phytochem. 2019; 163: 89-98.

3. Speranza J, Miceli N, Taviano MF, Ragusa S, Kwiecien I, Szopa A, et al. Isatis tinctoria L. (Woad): A review of its botany, ethnobotanical uses, phytochemistry, biological activities, and biotechnological studies. Plants (Basel). 2020;9:298

4. Peng JY, Fan GR, Wu YT. Isolation and purification of clemastanin B and indigoticoside A from Radix Isatidis by high-speed counter-current chromatography. J Chromatogr A. 2005; 1091: 89-93.

5. Kong WJ, Zhao YL, Shan LM, Xiao XH, Guo WY. Thermochemical studies on the quantity-antibacterial effect relationship of four organic acids from Radix Isatidis on Escherichia coli growth. Biol Pharm Bull. 2008; 31: 1301-5.

6. Tong C, Chen ZW, Liu F, Qiao YY, Chen T, Wang XB. Antiviral activities of Radix isatidis polysaccharide against pseudorabies virus in swine testicle cells. BMC Complement Med. 2020;20:48.

7. Chen JF, Dong X, Li Q, Zhou X, Gao SH, Chen RB, et al. Biosynthesis of the active compounds of Isatis indigotica based on transcriptome sequencing and metabolites profiling. BMC Genomics. 2013; 14: 857.

8. Liu BB, Sun GL. Transcriptome and miRNAs analyses enhance our understanding of the evolutionary advantages of polyploidy. Crit Rev Biotechnol. 2019; 39: 173-180.

9. Xu CC, Ge YM, Wang JB. Molecular basis underlying the successful invasion of hexaploid cytotypes of Solidago canadensis L.: Insights from integrated gene and miRNA expression profiling. Ecol Evol. 2019; 9: 4820-4852.

10. Renny-Byfield S, Wendel JF. Doubling down on genomes: polyploidy and crop plants. Am J Bot. 2014; 101: 1711-1725.

11. Yan H, Bombarely A, Xu B, Wu B, Frazier TP, Zhang X, et al. Autopolyploidization in switchgrass alters phenotype and flowering time via epigenetic and transcription regulation. J Exp Bot. 2019; 70: 5673-5686.

12. Martin SL, Husband BC. Adaptation of diploid and tetraploid Chamerion angustifolium to elevation but not local environment. Evolution. 2013; 67: 1780-1791.

13. Madlung A. Polyploidy and its effect on evolutionary success: old questions revisited with new tools. Heredity (Edinb). 2013; 110: 99-104.

14. Gao R, Wang HB, Dong B, Yang XD, Chen SM, Jiang JF, et al. Morphological, Genome and gene expression changes in newly induced autopolyploid Chrysanthemum lavandulifolium (Fisch. ex Trautv.) Makino. Int J Mol Sci. 2016; 17: 1690.

15. Gallagher JP, Grover CE, Hu G, Wendel JF. Insights into the ecology and evolution of polyploid plants through network analysis. Mol Ecol. 2016; 25(11): 2644-2660

16. Xiang ZX, Tang XL, Liu WH, Song CN. A comparative morphological and transcriptomic study on autotetraploid Stevia rebaudiana (bertoni) and its diploid. Plant Physiol Biochem. 2019; 143: 154-164.

17. Xiao Y, Feng JX, Li Q, Zhou YY, Bu QT, Zhou JH, et al. IMRRKY34 positively regulates yield, lignan biosynthesis and stress tolerance in Isatis indigotica. Acta Pharm Sin B. 2020; 10: 2417-2432.

18. Lu BB, Pan $X Z$, Zhang L, Huang BB, Sun LN, Li B, et al. A genome-wide comparison of genes responsive to autopolyploidy in Isatis indigotica using Arabidopsis thaliana Affymetrix genechips. Plant Mol Biol Rep. 2006; 24: 197-204.

19. Zhou YY, Kang L, Liao SY, Pan Q, Ge XH, Li ZY. Transcriptomic analysis reveals differential gene expressions for cell growth and functional secondary metabolites in induced autotetraploid of Chinese woad (Isatis indigotica Fort.). PLoS One. 2015; 10: e0116392.

20. Chinese Pharmacopoeia Commission. Pharmacopoeia of China 2020 Edition. Beijing: China Medical Science Press; pp. 22, 208, 214

21. Dong NQ, Sun Y, Guo T, Shi CL, Zhang YM, Kan Y, et al. UDPglucosyltransferase regulates grain size and abiotic stress tolerance associated with metabolic flux redirection in rice. Nat Commun. 2020; 11: 2629.

22. Yang BH, He S, Liu Y, Liu BC, Ju YL, Kang DZ, et al. Transcriptomics integrated with metabolomics reveals the effect of regulated deficit irrigation on anthocyanin biosynthesis in Cabernet Sauvignon grape berries. Food Chem. 2020; 314: 126170

23. Tan MP, Cheng D, Yang YN, Zhang GQ, Qin MJ, Chen J, et al. Co-expression network analysis of the transcriptomes of rice roots exposed to various cadmium stresses reveals universal cadmium-responsive genes. BMC Plant Biol. 2017; 17: 194.

24. Minghui K, Haolin W, Qiao Y, Li H, Quanjun H, Tao M, et al. A chromosomescale genome assembly of Isatis indigotica, an important medicinal plant used in traditional Chinese medicine: An Isatis genome. Hortic Res. 2020; 7: 18.

25. Kanehisa M. Toward understanding the origin and evolution of cellular organisms. Protein Sci. 2019; 28: 1947-1951.

26. Ramsey J, Ramsey TS. Ecological studies of polyploidy in the 100 years following its discovery. Philos Trans R Soc Lond B Biol Sci. 2014; 369: 20130352.

27. Jathish P, Armin S, Vasiliki Z, A. DM, Christin A, Regina F, et al. The trehalose 6-phosphate pathway impacts vegetative phase change in Arabidopsis thaliana. Plant J. 2020; 104: 768-780.

28. Li HW, Zang BS, Deng XW, Wang XP. Overexpression of the trehalose-6phosphate synthase gene OsTPS1 enhances abiotic stress tolerance in rice. Planta. 2011; 234: 1007-1018.

29. Dios BJd, Arjun T, Xavier Z, W SM, Jesús P, Matleena P, et al. EARLY RESPONSE TO DEHYDRATION 7 remodels cell membrane lipid composition during cold stress in Arabidopsis. Plant Cell physiol. 2020; 62: 80-91.

30. Zhang X, Yang ZH, Wu DS, Yu F. RALF-FERONIA signaling: Linking plant immune response with cell growth. Plant Commun. 2020; 1: 100084.

31. Wang L, Yang T, Wang BQ, Lin QL, Zhu SR, Li CY, et al: RALF1-FERONIA complex affects splicing dynamics to modulate stress responses and growth in plants. Sci Adv. 2020; 6: eaaz1622.

32. Jiang $Q$, Ma XJ, Gong XD, Zhang $J H$, Teng $S$, Xu JL, et al. The rice OsDG2 encoding a glycine-rich protein is involved in the regulation of chloroplast development during early seedling stage. Plant Cell Rep. 2014; 33: 733-44.

33. Zhu SR, Pacheco JM, Estevez JM, Yu F. Autocrine regulation of root hair size by the RALF FERONIA RSL4 signaling pathway. New Phytol. 2020; 227: 45-49.

34. He YX, Xu J, Wang XY, He XM, Wang YXY, Zhou JG, et al. The Arabidopsis pleiotropic drug resistance transporters PEN3 and PDR12 mediate camalexin secretion for resistance to Botrytis cinerea. Plant cell. 2019; 31: 2206-2222.

35. Andreas M, Christoph B, Lennart EL, Bernhard W, Ulrike S, Stefanie D, et al. A substrate of the $A B C$ transporter PEN3 stimulates bacterial flagellin (flg22)induced callose deposition in Arabidopsis thaliana. J Biol Chem. 2019; 294: 6857-6870.

\section{Publisher's Note}

Springer Nature remains neutral with regard to jurisdictional claims in published maps and institutional affiliations.

Ready to submit your research? Choose BMC and benefit from:

- fast, convenient online submission

- thorough peer review by experienced researchers in your field

- rapid publication on acceptance

- support for research data, including large and complex data types

- gold Open Access which fosters wider collaboration and increased citations

- maximum visibility for your research: over $100 \mathrm{M}$ website views per year

At BMC, research is always in progress.

Learn more biomedcentral.com/submissions 